\title{
von Willebrand Factor Gene Expression in Primary Lower Grade Glioma: Mutually Co-Occurring Mutations in von Willebrand Factor, ATRX, and TP53
}

\author{
Steven Lehrer ${ }^{1}$, Peter H. Rheinstein ${ }^{2}$, Sheryl Green ${ }^{1}$, Kenneth E. Rosenzweig ${ }^{1}$ \\ 'Department of Radiation Oncology, Icahn School of Medicine at Mount Sinai, New York, NY, USA \\ 2Severn Health Solutions, Severna Park, MD, USA
}

Received August 3, 2018

Revised September 30, 2018

Accepted December 10, 2018

\section{Correspondence}

Steven Lehrer

Box 1236 Radiation Oncology,

Mount Sinai Medical Center,

1 Gustave L. Levy Place,

New York, NY 10029, USA

Tel: $+1-212-765-7132$

Fax: +1-212-245-9708

E-mail: steven.lehrer@mssm.edu
Background Venous thromboembolism is a common complication in patients with glioma. The clotting factor von Willebrand factor (NWF) is a highly adhesive procoagulant molecule that mediates platelet adhesion to endothelial and subendothelial surfaces. In the current analysis, we examined The Cancer Genome Atlas (TCGA) data to assess the WWF gene in patients with lower grade gliomas.

Methods For newly diagnosed gliomas, we evaluated the association between WWF and overall survival in the Genomic Data Commons TCGA Lower Grade Glioma (LGG) dataset in TCGA. Simple statistics were calculated to identify patterns of mutual exclusivity or co-occurrence of WWF mutations. For each pair of query genes an odds ratio was calculated that indicates the likelihood that the mutations in the two genes are mutually exclusive or co-occurrent across the selected cases. To determine whether the identified relationship was significant for a gene pair, Fisher's exact test was performed.

Results Lower grade gliomas with less VWF gene expression had significantly better survival than those with more WWF gene expression (hazard ratio $0.64,95 \%$ confidence interval 0.44 to 0.92 , $p=0.015$ log rank test). When we analyzed the data with Cox regression, WWF expression had a significant effect on survival $(p=0.02)$ that was unrelated to the effect of IDH1 expression $(p=0.062)$, TP53 expression $(p=0.135)$, independent of ATRX expression $(p=0.021)$ and histology (astrocytoma versus oligoastrocytoma and oligodendroglioma, $p=0.002$ ). WWF mutations significantly co-occur with mutations in TP53 and ATRX $(p<0.001)$.

Conclusion The deleterious prognostic effect of WWF expression and its co-occurrent mutations with TP53 and ATRX in lower grade gliomas are not surprising, given WWF's role in other cancers. Therefore, WWF gene expression may be a clinically important risk marker in lower grade glioma.

Key Words Glioma; Glioblastoma; Survival; von Willebrand factor; The Cancer Genome Atlas.

\section{INTRODUCTION}

Malignant brain tumors are accompanied by hypercoagulability. Glioblastoma multiforme (GBM), the most common primary tumor of the central nervous system (CNS), is associated with heightened coagulation and venous thromboem-

This is an Open Access article distributed under the terms of the Creative Commons Attribution Non-Commercial License (https://creativecommons.org/licenses/by-nc/4.0) which permits unrestricted non-commercial use, distribution, and reproduction in any medium, provided the original work is properly cited.

Copyright (c) 2019 The Korean Brain Tumor Society, The Korean Society for NeuroOncology, and The Korean Society for Pediatric Neuro-Oncology bolism (VTE). The circulating substance inducing the hypercoagulability is uncertain. Anticoagulants are frequently administered to GBM patients, although these agents increase the risk of intracerebral hemorrhage [1].

VTE is a common complication in patients with glioma [2]. VTE may be a prognostic factor for these patients [3].

Thrombin, an enzyme in blood plasma, causes the clotting of blood by converting fibrinogen to fibrin. In lower grade gliomas, thrombin has multiple effects. Intracerebral infusion of argatroban, a specific thrombin inhibitor, reduces brain edema and neurologic deficits in a rat glioma model. Thrombin im- 
munoreactivity is found in cultured rat glioma cells and human glioblastoma. In addition, thrombin induces glioma cell proliferation in vitro; whereas argatroban reduces glioma mass [4].

Another clotting factor, von Willebrand factor (VWF), is a highly adhesive procoagulant molecule that mediates platelet adhesion to endothelial and subendothelial surfaces. Normally VWF is expressed exclusively in endothelial cells and megakaryocytes. However, a few studies have reported VWF detection in cancer cells of non-endothelial origin, including osteosarcoma and glioma [5]; and VWF is a prognostic factor in GBM [6].

In the current analysis, we examined The Cancer Genome Atlas (TCGA) data to assess the VWF gene in patients with lower grade gliomas.

\section{MATERIALS AND METHODS}

For newly diagnosed gliomas, we evaluated the association between VWF and overall survival in the Genomic Data Commons (GDC) TCGA Lower Grade Glioma (LGG) dataset in TCGA. To access and analyze the data we used:

- GDC Data Portal (https://portal.gdc.cancer.gov/);

- UCSC Xena browser (https://xenabrowser.net);

- Oncomine (https://oncomine.org);

- cBioPortal (http://cbioportal.org).

Survival data of the glioma subgroup were extracted for analysis and generation of Kaplan-Meier curves for overall survival. The optimal cutoff was identified by methods described in the R2 web-based application (http://r2.amc.nl); the method divides the sample, ascending order gene expression, into equal-sized groups. Further details have been published [7].

Gene expression is quantitated as Fragments Per Kilobase of transcript per Million mapped reads upper quartile (fpkmuq), which is an RNA-Seq-based expression normalization method [8].

Simple statistics were calculated to identify patterns of mutual exclusivity or co-occurrence of mutations. For each pair of query genes (e.g., VWF and ATRX), an odds ratio (OR) is calculated (Equation 1) that indicates the likelihood that the mutations in the two genes are mutually exclusive or co-occurrent across the selected cases:

$$
\mathrm{OR}=(\mathrm{A} * \mathrm{D}) /(\mathrm{B} * \mathrm{C}),
$$

where $\mathrm{A}=$ number of cases altered in both genes; $\mathrm{B}=$ number of cases altered in VWF but not ATRX; $\mathrm{C}=$ number of cases altered in ATRX but not VWF; and $\mathrm{D}=$ number of cases altered in neither gene. Each pair was then assigned to one of five categories indicative of a tendency toward mutual exclusivity, of a tendency toward co-occurrence, or of no association. To determine whether the identified relationship is significant for a gene pair, Fisher's exact test was performed [9].

\section{RESULTS}

We analyzed data from 513 patients with primary lower grade glioma. The patients' mean age was $43 \pm 14$ years (mean \pm SD). $55 \%$ of the patients were male, $45 \%$ female. $92.1 \%$ were white, $4.1 \%$ African-American, $1.6 \%$ Asian, $0.2 \%$ American Indian or Alaska native, $1.9 \%$ unclassified. Mean survival was 7.8 years. Histology and histologic grade are shown in Table 1. Molecular subtype information [10] is in Fig. 1.

VWF was significantly overexpressed in lower grade gliomas, especially in astrocytomas $(p=0.008, t=2.471$, Fold Change: 1.084, data from RefSeq Genes, UCSC refGene, July 2009, hg18, NCBI 36.1, March 2006) (Fig. 2).

Histology of tumors and VWF expression in the current

Table 1. Histology and histologic grade of patients studied

\begin{tabular}{lc}
\hline \multicolumn{1}{c}{ Histology } & No. of patients \\
\hline Astrocytoma & 194 \\
Oligodendroglioma & 189 \\
Oligoastrocytoma & 130 \\
Histologic grade & \\
Grade 3 & 264 \\
Grade 2 & 248 \\
NOS & 1 \\
\hline
\end{tabular}

NOS, not otherwise specified

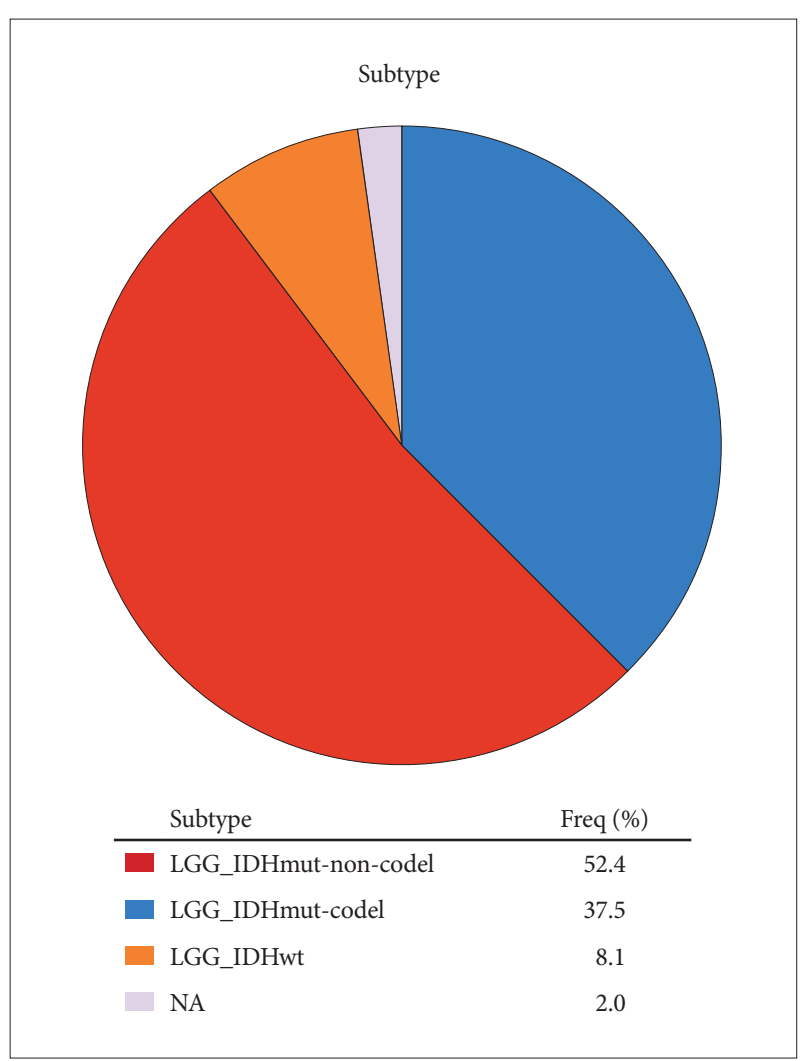

Fig. 1. Molecular subtypes of tumors studied. LGG, Lower Grade Glioma; IDH, isocitrate dehydrogenase; NA, not analyzed. 
analysis are listed in Table 2. There was significant variability of VWF expression in astrocytomas, oligodendrogliomas and oligoastrocytomas ( $p=0.045$, one way analysis of variance). VWF was most often mutated and amplified in diffuse gliomas (grade II gliomas, also known as diffuse low-grade glioma) (Fig. 3) [11]. A previous study suggested that percentage of VWF expressing tumor cells is higher in GBM and osteosarcoma than astrocytic tumors [5].

Survival of patients with astrocytomas was significantly worse than survival of patients with oligodendroglioma or oligoastrocytoma ( $p=0.0064$, log rank test) (Fig. 4$)$. Note that in the 2016 CNS WHO classifications, oligodendrogliomas must carry both an isocitrate dehydrogenase (IDH) mutation and a $1 \mathrm{p} / 19 \mathrm{q}$ codeletion. Gliomas with an IDH mutation but intact $1 \mathrm{p} / 19 \mathrm{q}$ genomic regions are classified as astrocytomas, as are those that lack an IDH mutation [12,13]. Much of the TCGA data we present and analyze here was collected before the 2016 WHO classification change.

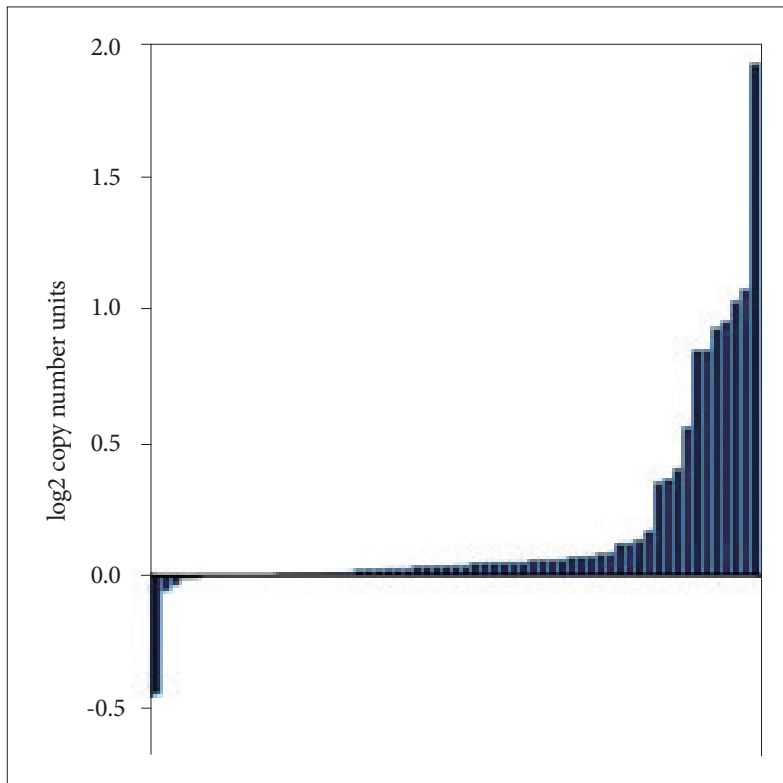

Fig. 2. Expression of VWF in 63 astrocytomas (TCGA-DU-729901A-21D-2023-01). VWF is significantly overexpressed $(p=0.008$, $t=2.471$, Fold Change: 1.084). RefSeq Genes, UCSC refGene, July 2009, hg18, NCBI 36.1, March 2006. The horizontal axis indicates specimen number (1-63). VWF, von Willebrand factor.

Table 2. Histology and VWF gene expression (fpkm-uq) of 510 primary tumors in this study

\begin{tabular}{lcc}
\hline & $\mathrm{n}$ & $\mathrm{VWF}$ \\
\hline Astrocytoma & 192 & $17.8 \pm 0.07$ \\
Oligoastrocytoma & 128 & $17.5 \pm 1.00$ \\
Oligodendroglioma & 190 & $17.6 \pm 1.00$ \\
\hline
\end{tabular}

Variability of gene expression was significant ( $p=0.045$, one way analysis of variance). VWF, von Willebrand factor; fpkm-uq, Fragments Per Kilobase of transcript per Million mapped reads upper quartile
Survival versus VWF gene expression is shown in Fig. 5. Lower grade gliomas with less VWF gene expression had significantly better survival than those with more VWF gene expression (hazard ratio $0.64,95 \%$ confidence interval 0.44 to $0.92, p=0.015 \log$ rank test). The effect of VWF gene expression on survival was even more evident when the sample was analyzed as three groups ( $p=0.00019$ ).

IDH1, TP53 and ATRX mutations are present in $40 \%$ or more adult lower grade gliomas [14-16]. Among these tumors, as noted above, patients with astrocytomas have poorer survival than patients with oligoastrocytomas or oligodendrogliomas; prognosis of oligoastrocytomas or oligodendrogliomas is approximately equivalent. When we analyzed the data with Cox regression, VWF expression had a significant effect on survival $(p=0.02)$ that was unrelated to the effect of IDH1 expression

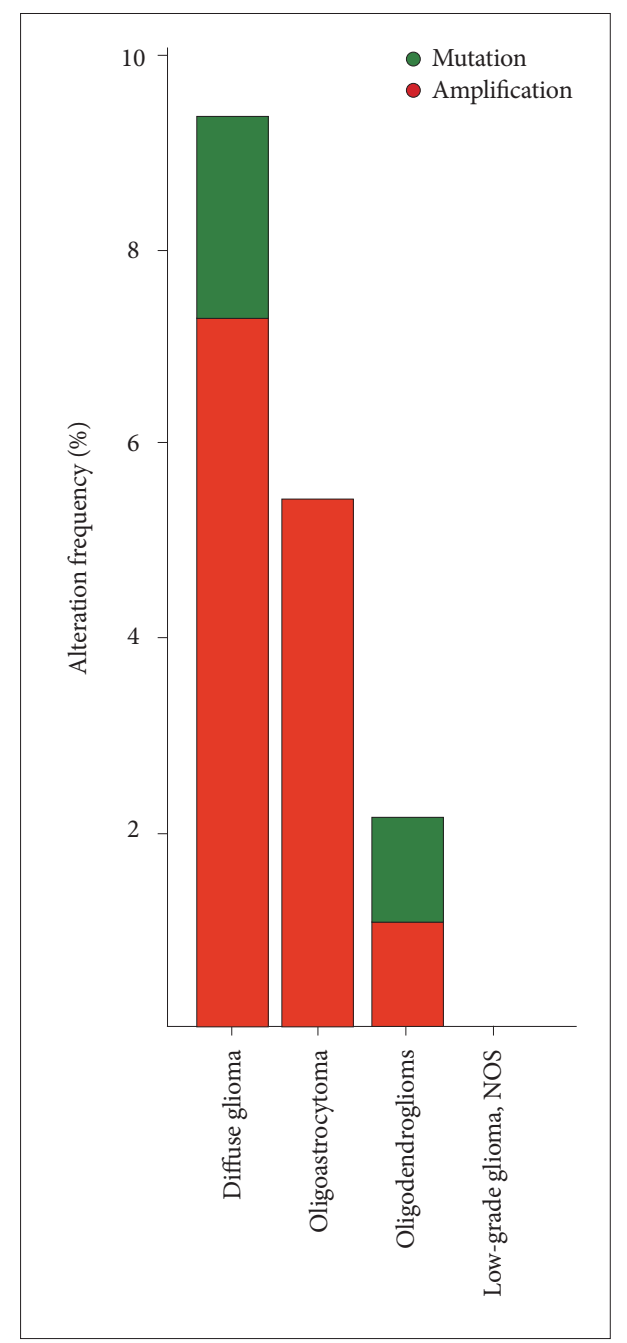

Fig. 3. VWF was most often mutated and amplified in diffuse gliomas (http://cbioportal.org). Diffuse gliomas are classified according to the 2016 WHO system by both histologic and molecular characteristics as IDH-mutant or IDH-wildtype astrocytomas; IDHmutant and 1p19q-codeleted oligodendrogliomas; and IDH-mutant or IDH-wildtype glioblastomas. VWF, von Willebrand factor; WHO, World Health Organization; IDH, isocitrate dehydrogenase. 
( $p=0.062)$, TP53 expression $(p=0.135)$, independent of ATRX expression ( $p=0.021$ ) and histology (astrocytoma versus oligoastrocytoma and oligodendroglioma, $p=0.002$ ).

VWF mutations significantly co-occur with mutations in TP53 and ATRX $(p<0.001)$ (Table 3, Fig. 6). The gene pair

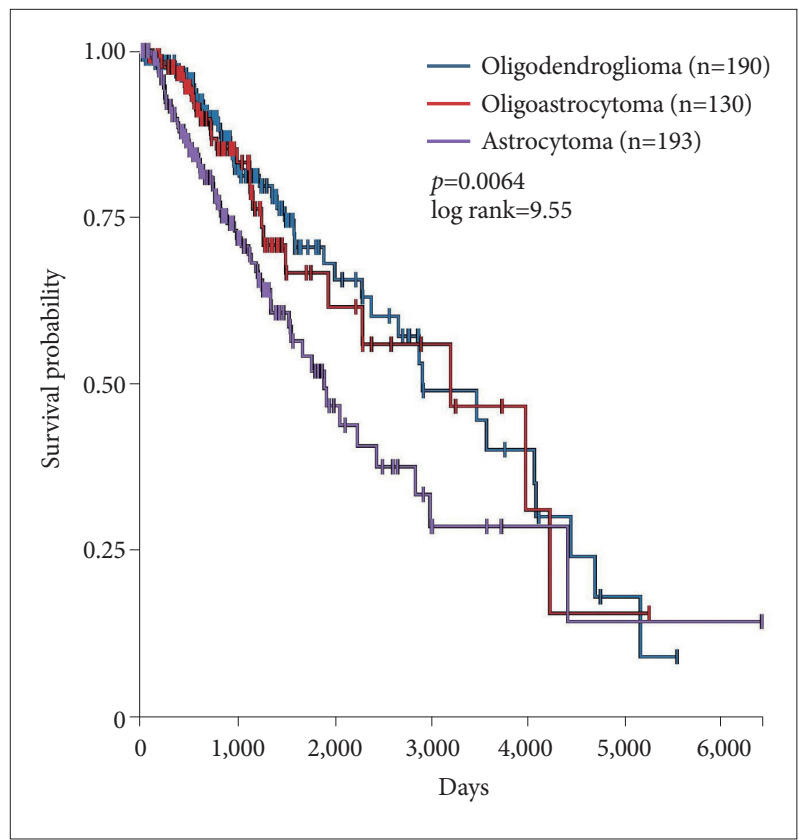

Fig. 4. Survival of lower grade glioma by histology in 513 patients. Survival of astrocytomas was significantly worse than oligodendroglioma or oligoastrocytoma ( $p=0.0064$, log rank test).
TP53/ATRX had 191 significantly co-occurrent alterations; the pair VWF/TP53 had 26 significantly co-occurrent alterations; the pair VWF/ATRX had 23 significantly co-occurrent alterations.

VWF is not an enzyme and its primary function is binding to other proteins, in particular Factor VIII. In addition, the destruction of VWF is largely mediated by the enzyme ADAMTS 13. Therefore, to address the effects of elevated VWF expression on the pathogenesis of thrombosis in lower grade glioma, we examined the expression of the related genes for Factor VIII and ADAMTS13. We found no correlation with VWF.

Lower grade gliomas include various types of tumors. IDH1 is the most frequently mutated gene, in about $80 \%$ of cases. IDH1 versus VWF mRNA expression by mutation status is shown in Fig. 7. The correlation is significant. Tumor grade and VWF mRNA expression by mutation status is shown in Fig. 8; grade 3 tumors have more mutations than grade 2 .

\section{DISCUSSION}

Lower grade glioma is a uniformly fatal disease of young adults with survival averaging approximately 7 years [17]. Although lower grade glioma patients have better survival than patients with high grade (WHO grade III/IV) glioma, almost all lower grade gliomas eventually progress to high grade glioma and death. Data from the Surveillance, Epidemiology and End Results (SEER) program of the National Cancer Institute
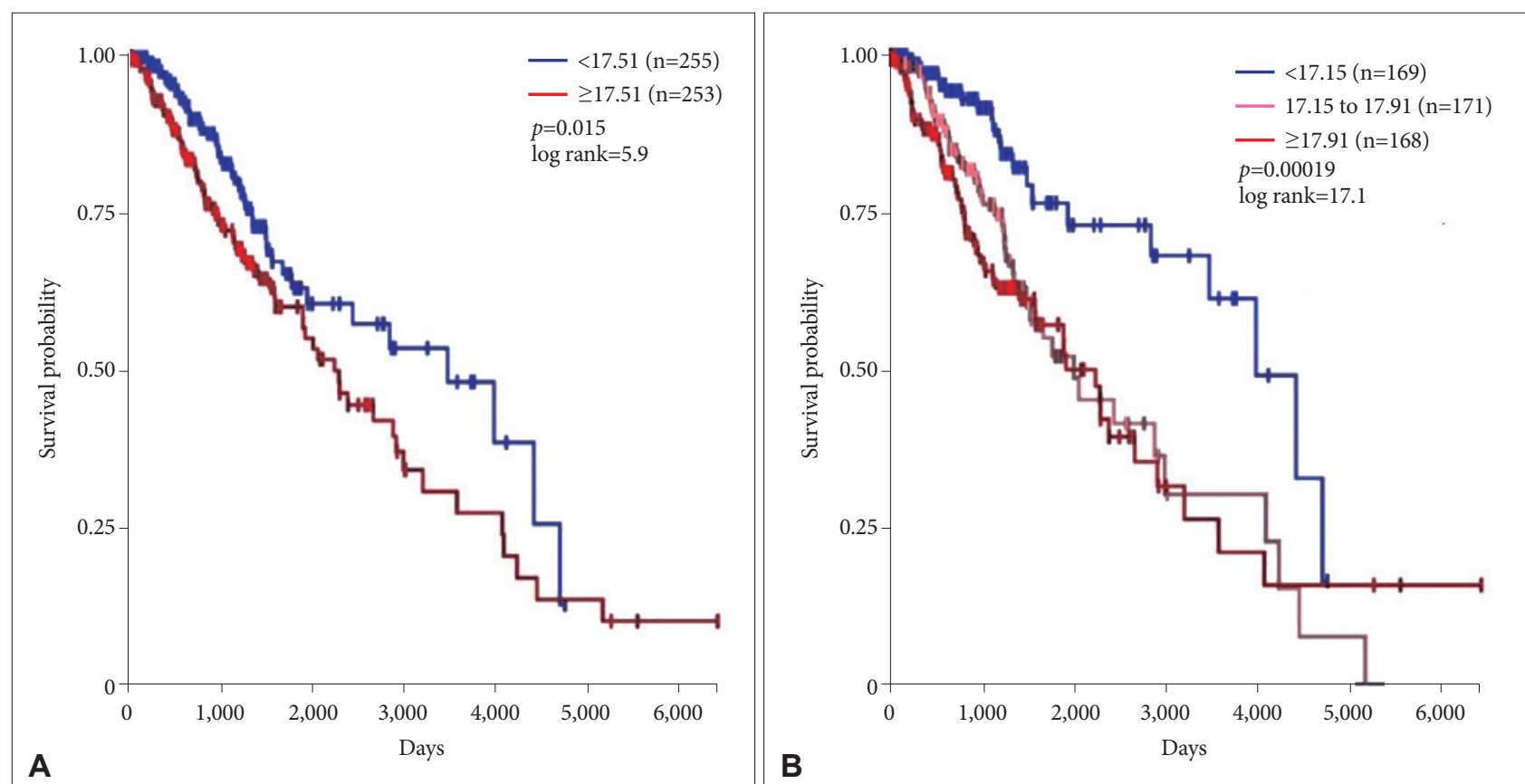

Fig. 5. Survival versus VWF gene expression in 508 patients. A: Lower grade gliomas with less VWF gene expression had significantly better survival than those with more VWF gene expression (hazard ratio $0.64,95 \%$ confidence interval 0.44 to $0.92, p=0.015$ log rank test). B: The effect of VWF gene expression on survival was even more evident when the sample was analyzed as three groups $(p=0.00019)$. No VWF expression data were available for 5 of 513 patients analyzed in Fig. 3. VWF, von Willebrand factor. 
Table 3. The gene pair TP53/ATRX had 191 significantly co-occurrent alterations; the pair VWF/TP53 had 26 significantly co-occurrent alterations; the pair VWF/ATRX had 23 significantly co-occurrent alterations

\begin{tabular}{cccccccccc}
\hline Gene A & Gene B & Neither & A Not B & B Not A & Both & Log odds ratio & $p$-value & Adjusted $p$-value & Tendency \\
\hline TP53 & ATRX & 243 & 58 & 15 & 191 & $>3$ & $<0.001$ & $<0.001$ & Co-occurrence \\
VWF & TP53 & 255 & 3 & 223 & 26 & 2.294 & $<0.001$ & $<0.001$ & Co-occurrence \\
VWF & ATRX & 295 & 6 & 183 & 23 & 1.821 & $<0.001$ & $<0.001$ & Co-occurrence \\
\hline
\end{tabular}

$p$ value was Bonferroni adjusted. VWF, von Willebrand factor

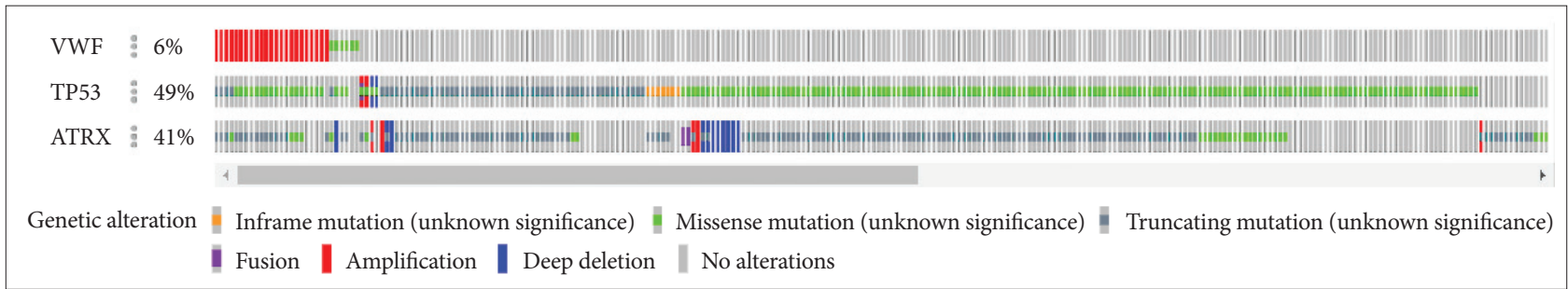

Fig. 6. Diagrammatic representation of significantly co-occurring mutations in VWF/ATRX, VWF/TP53 and TP53/ATRX. cDNA was altered in $266(52 \%)$ of 507 sequenced cases/patients (507 total) (cBioportal.org). VWF, von Willebrand factor.

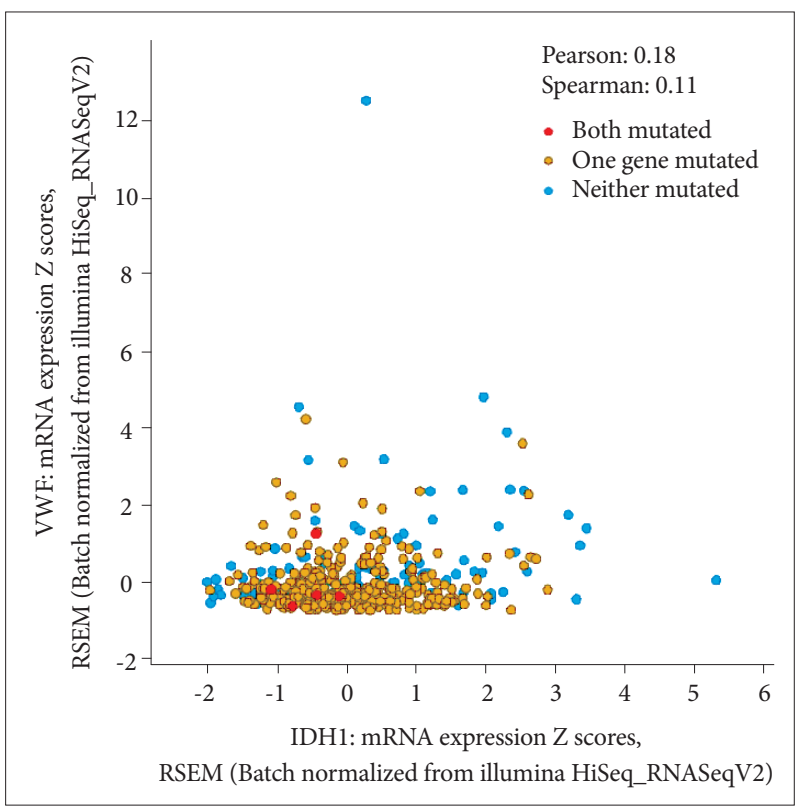

Fig. 7. IDH1 versus VWF mRNA expression by mutation status. The correlation is significant $(p<0.05)$. VWF, von Willebrand factor; $\mathrm{IDH}$, isocitrate dehydrogenase.

suggest that for the majority of lower grade glioma patients, overall survival has not significantly improved over the past three decades [17].

Multiple prognostic factors have been described: Tumor histology, grade, location, contrast enhancement, patient age, performance status, and seizure history [18]. Survival probability in patients with a glioma having a high median Tumor Blood Flow (mTBF) has been shown to be lower than in patients with a glioma having a low mTBF [19]. Recently, tumor genome has proven to be a prognostic factor [14,15], as well as F13A1 copy number segments [20].

Armand Trousseau first reported the relationship of malig-

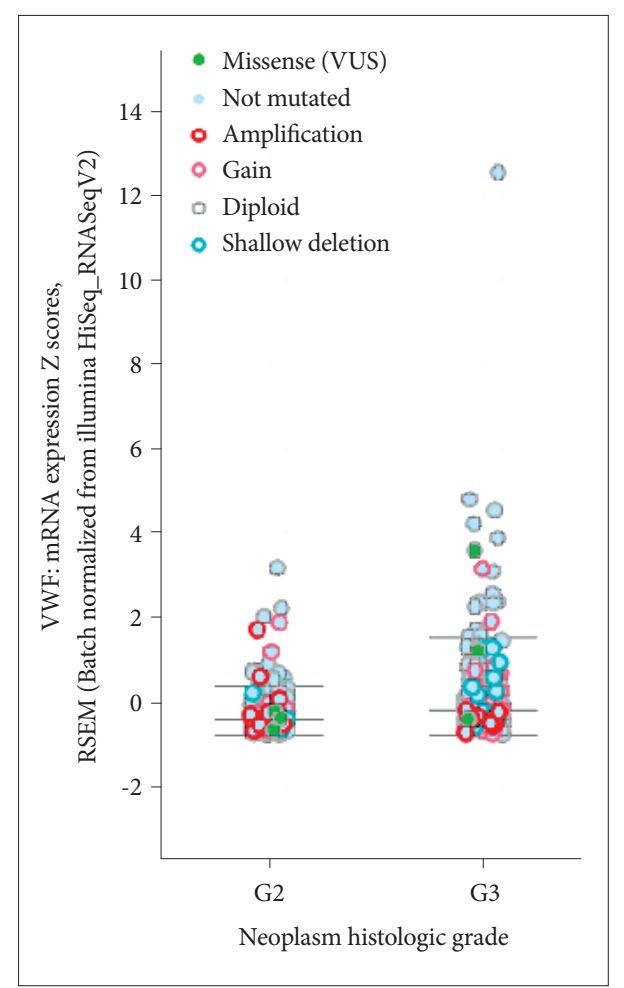

Fig. 8. Histologic grade and VWF mRNA expression by VWF mutation status. VWF, von Willebrand factor; VUS, variants of uncertain significance.

nant tumors and coagulation in 1865 . Trousseau diagnosed the syndrome in himself two years later, dying soon afterward of gastric cancer. Recent findings suggest that genetic pathways within tumor cells might trigger thrombotic phenomena, worsening prognosis [21].

Biological processes or pathways in cancer are often deregulated through different genes or by multiple different mechanisms. But cancer gene mutations usually do not occur at 
random. Mutations of certain cancer genes tend to co-occur, indicating that they may work in tandem to drive tumor formation and development [9]. This appears to be the case with the co-occurring VWF, ATRX, and TP53 mutations.

The deleterious prognostic effect of VWF expression in lower grade gliomas is not surprising, given its role in other cancers. VWF fibers promote cancer-associated platelet aggregation in malignant melanoma of mice and humans [22]; and cancer cell-derived VWF enhances metastasis of gastric adenocarcinoma [23].

An analysis of lower grade glioma in TCGA has shown IDH, $1 \mathrm{p} / 19 \mathrm{q}$, and TP53 status to be more indicative of prognosis than histologic class. Moreover, lower-grade gliomas with an IDH mutation either had $1 \mathrm{p} / 19 \mathrm{q}$ codeletion or carried a TP53 mutation. Most lower-grade gliomas without an IDH mutation were molecularly and clinically similar to glioblastoma [16].

VWF gene expression may be another clinically important prognostic marker in lower grade glioma. The risk of VTE in glioma patients is high, and extends beyond the postoperative period. The procoagulant molecule tissue factor appears to play an important role, but other markers are needed [24]. VWF may represent a new marker. Though thromboembolic events lead to worse outcomes, risks of anticoagulant administration should be better defined. Further studies of VWF would be worthwhile.

\section{Conflicts of Interest}

The authors have no potential conflicts of interest.

\section{REFERENCES}

1. Norden AD, Bartolomeo J, Tanaka S, et al. Safety of concurrent bevacizumab therapy and anticoagulation in glioma patients. J Neurooncol 2012;106:121-5.

2. Al Megren M, De Wit C, Al Qahtani M, Le Gal G, Carrier M. Management of venous thromboembolism in patients with glioma. Thromb Res 2017;156:105-8.

3. Simanek R, Vormittag R, Hassler M, et al. Venous thromboembolism and survival in patients with high-grade glioma. Neuro Oncol 2007;9: 89-95.

4. Hua Y, Tang L, Keep RF, et al. The role of thrombin in gliomas. J Thromb Haemost 2005;3:1917-23.

5. Mojiri A, Stoletov K, Carrillo MA, et al. Functional assessment of von Willebrand factor expression by cancer cells of non-endothelial origin. Oncotarget 2017;8:13015-29.

6. Marfia G, Navone SE, Fanizzi C, et al. Prognostic value of preoperative von Willebrand factor plasma levels in patients with glioblastoma. Cancer Med 2016;5:1783-90.

7. R2 Support Team. Working with Kaplan Meier. In: R2 Tutorials. Release 3.1.2. Amsterdam: Jan Koster (Academic Medical Center);2017. p.73-82.

8. Shahriyari L. Effect of normalization methods on the performance of supervised learning algorithms applied to HTSeq-FPKM-UQ data sets: 7SK RNA expression as a predictor of survival in patients with colon adenocarcinoma. Brief Bioinform 2017 Nov 3 [Epub]. https:// doi.org/10.1093/bib/bbx153.

9. Gao J, Aksoy BA, Dogrusoz U, et al. Integrative analysis of complex cancer genomics and clinical profiles using the cBioPortal. Sci Signal 2013;6:pl1.

10. Ceccarelli M, Barthel FP, Malta TM, et al. Molecular profiling reveals biologically discrete subsets and pathways of progression in diffuse glioma. Cell 2016;164:550-63.

11. Wang S, Jin F, Fan W, et al. Gene expression meta-analysis in diffuse low-grade glioma and the corresponding histological subtypes. Sci Rep 2017;7:11741.

12. Louis DN, Perry A, Reifenberger G, et al. The 2016 World Health Organization Classification of Tumors of the Central Nervous System: a summary. Acta Neuropathol 2016;131:803-20.

13. DeWeerdt $S$. The genomics of brain cancer. Nature 2018;561:S54-5.

14. Venneti S, Huse JT. The evolving molecular genetics of low-grade glioma. Adv Anat Pathol 2015;22:94-101.

15. Buckner J, Giannini C, Eckel-Passow J, et al. Management of diffuse lowgrade gliomas in adults - use of molecular diagnostics. Nat Rev Neurol 2017;13:340-51.

16. Cancer Genome Atlas Research Network, Brat DJ, Verhaak RG, Aldape $\mathrm{KD}$, et al. Comprehensive, integrative genomic analysis of diffuse lower-grade gliomas. N Engl J Med 2015;372:2481-98.

17. Claus EB, Walsh KM, Wiencke JK, et al. Survival and low-grade glioma: the emergence of genetic information. Neurosurg Focus 2015;38:E6.

18. Stupp R, Janzer RC, Hegi ME, Villemure JG, Mirimanoff RO. Prognostic factors for low-grade gliomas. Semin Oncol 2003;30(6 Suppl 19): 23-8.

19. Furtner J, Bender B, Braun C, et al. Prognostic value of blood flow measurements using arterial spin labeling in gliomas. PLoS One 2014;9: e99616.

20. Lehrer S, Dembitzer FR, Rheinstein PH, Rosenzweig KE. In primary glioblastoma fewer tumor copy number segments of the F13A1 gene are associated with poorer survival. Thromb Res 2018;167:12-14.

21. Varki A. Trousseau's syndrome: multiple definitions and multiple mechanisms. Blood 2007;110:1723-9.

22. Bauer AT, Suckau J, Frank K, et al. von Willebrand factor fibers promote cancer-associated platelet aggregation in malignant melanoma of mice and humans. Blood 2015;125:3153-63.

23. Yang AJ, Wang M, Wang Y, et al. Cancer cell-derived von Willebrand factor enhanced metastasis of gastric adenocarcinoma. Oncogenesis 2018;7:12.

24. Jenkins EO, Schiff D, Mackman N, Key NS. Venous thromboembolism in malignant gliomas. J Thromb Haemost 2010;8:221-7. 